\title{
Ileosigmoid knot as a cause of acute abdomen at 28 weeks of pregnancy: A rare case report
}

\author{
Osman Toktaş ${ }^{1^{*}}$ and Numan Çim ${ }^{2}$ \\ ${ }^{1} \mathrm{~V}$ an Yuzuncu Yil University, Medical Faculty, Department of General Surgery, Van, Turkey \\ ${ }^{2} V$ an Yuzuncu Yil University, Medical Faculty, Department of Gynaecology, Van, Turkey
}

\begin{abstract}
Ileosigmoid knot (ISK), also known as compound volvulus or double volvulus, is an unusual surgical emergency and is a rare condition leading to intestinal obstruction. ISK is an unusual phenomenon in the West but is relatively more common in some African, Asian, and Middle Eastern nations. This condition is serious, usually progressing rapidly to gangrene. Awareness of the condition is vital for the early diagnosis and optimal management of the patients. ISK should be considered in the differential diagnosis of patients presenting with ileus. In this report, we present a rare case of ISK in a 41-year-old, 28-week pregnant woman whose diagnosis was established intraoperatively.
\end{abstract}

Key Words: Ileosigmoid knot, ileus, small bowel obstruction

\section{Introduction}

Ileosigmoid knot (ISK) is an unusual clinical condition of small bowel obstruction in which the ileum twists around the base of the sigmoid colon and forms a pseudo-knot (1). Turkey, particularly Eastern Anatolia, where our hospitals are located, has a high incidence of this otherwise rare disease (2). The strangulation caused by the "knot" threatens the viability of both ileum and sigmoid colon (3). In severe cases, it can cause gangrene of both the small bowel and the sigmoid colon. Preoperative diagnosis of this condition is difficult due to its rarity and atypical radiographic findings (4).

In recent years, computed tomography (CT) has emerged as a preoperative diagnostic tool. Generalized peritonitis and sepsis are the main causes of the poor outcome in patients with ISK. After hemodynamic stabilization, immediate surgical intervention is the only viable option in these patients (5). Treatment of ISK consists of early and effective resuscitation followed by emergency surgery, the purpose of which is to release the knot, resect the gangrenous bowel segment, resume intestinal continuity, and prevent recurrence (2). In this report, we present an unusual case of ISK in a patient who was in her late pregnancy and underwent emergency surgery for acute abdomen.

\section{Case report}

A 41-year-old woman at 28 weeks of pregnancy with a history of chronic constipation presented to our clinic with severe lower abdominal pain of 12hour duration associated with progressive abdominal distension and vomiting. She had no co-morbidities and had no history of surgery.

On admission, her general status was moderate. Physical examination revealed a body temperature of $38.7^{\circ} \mathrm{C}$, blood pressure of $90 / 60 \mathrm{~mm} \mathrm{Hg}$, and heart rate of 105 per minute. Abdominal examination revealed diffuse abdominal distension, tenderness, and guarding. Digital rectal examination was normal.

Laboratory data showed a white cell count of 25,300/mm3 (normal range, 4,000-11,000/mm3), C-reactive protein $99 \mathrm{mg} / \mathrm{dL}$ (normal range, 3-5 $\mathrm{mg} / \mathrm{dL}$ ), and creatine kinase $618 \mathrm{u} / \mathrm{L}$ (normal range, 26-140 u/L). Kidney function tests and serum amylase levels were within normal limits.

Radiography and abdominal CT scans were not possible since the patient was pregnant; therefore, only abdominal ultrasonography (USG) was carried out under emergency conditions. Abdominal USG revealed $\sim 4.5 \mathrm{~cm}$ free liquid in the deepest part of the abdomen, dilated bowel loops, and dense content (ileus?). Gestational age was $28 \mathrm{w} 4 \mathrm{~d}$, the amniotic fluid was sufficient, the 
foetus was in head-first presentation, and fetal heartbeat was positive.

The patient was resuscitated with IV fluids and commenced on IV cefuroxime and metronidazole. A written consent was obtained from the patient at this stage. After adequate resuscitation, an exploratory laparotomy was performed. The peritoneal cavity contained about a litre of foulsmelling dark fluid. The mesentery of the ileum was also twisted around the ileum resulting in gangrene of the distal $10 \mathrm{~cm}$ of the ileum up to the ileocecal junction. The ileum was with a $360^{\circ}$ anticlockwise twist around the sigmoid colon (Figure 1 and 2). Following a $150-\mathrm{cm}$ resection of the gangrenous ileum and sigmoid, end-to-end ileo-ileal and colo-colic anastomoses were done. No ostomy was performed due to pregnancy. The bowel end was nonoedematous. A gynaecological consultation was performed intraoperatively but the caesarean section was not considered since it was too early.

Postoperative biliary drainage was $\sim 100-300 \mathrm{cc}$, and fetal heartbeat was positive. At postoperative day 3 , a gynaecological examination was performed by USG. Fetal heartbeat was negative. A caesarean section was performed due to fetal death, and ileal anastomotic leak was observed. Intraoperative cleaning and an end ileostomy were performed. The patient was uneventfully discharged at postoperative day 12. Ileostomy closed spontaneously at month 3 .

\section{Discussion}

Ileosigmoid knot (ISK), also known as double volvulus or volvulus ileo-sigmoid or ileo-sigmoid node, is a "node" formed by a volvulus of two intestinal segments: sigmoid colon and small

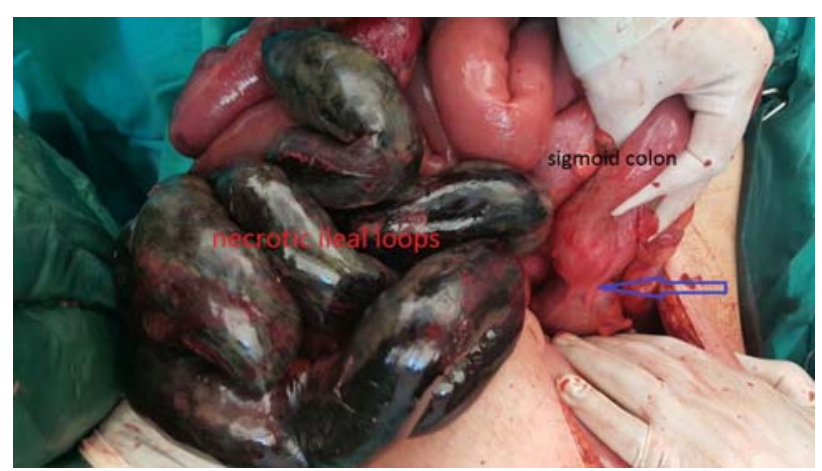

Fig. 1. Intraoperative appearance of the case, following the unravelling of the knot, indicates viable sigmoid colon and gangrenous small intestine. (Arrow indicates the base of the sigmoid) intestine, notably the ileum. ISK is a rare cause of intestinal obstruction that rapidly turns into gangrene of the ileum and the sigmoid colon (6).

This condition was first described by Riverius in the $16^{\text {th }}$ century (7). ISK is associated with $0.5 \%$ to $1.7 \%$ of intestinal obstructions, and the reported mortality rate varies from $0 \%$ to $48 \%$ (mean, 35.5\%). The mortality rate of ISK with bowel gangrene is known to be as high as $20 \%$ to $100 \%(8)$. The mortality rates are often related to the duration of the symptoms, presence or absence of gangrene, and general status of the patient involving the presence of septicemic shock (9). ISK is mostly seen in male patients (80.2\%) with a mean age of 40 years (range, $4-90$ years) (10). Our patient was a 41-year-old female in 28 weeks of pregnancy.

Subsequent peristalsis results in the formation of an ISK with an obstruction of 2 closed loops: one in the small intestine and the other in the sigmoid colon. Anatomic factors are also known to be responsible for ISK, including a long small bowel mesentery and freely mobile small bowel, and a long sigmoid colon on a narrow pedicle (11).

Late pregnancy is a predisposing risk factor for ISK due to the obvious displacement of the bowel (8). Therefore, we consider that the ISK in our patient was associated with the displacement of the bowel resulting from an enlarged uterus.

Ileosigmoid knot has three distinct types: Type I, the ileum (active component) wraps itself around the sigmoid colon (passive component) in the clockwise or anticlockwise direction (type A when clockwise and type B when anticlockwise). Type II, the sigmoid colon (active component) wraps itself around a loop of the ileum (passive component) in the clockwise or anticlockwise direction. Type III, the ileocecal segment (active

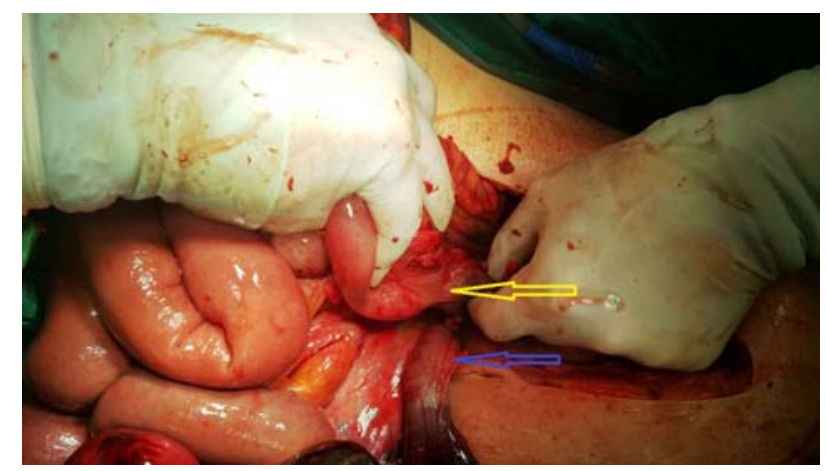

Fig. 2. Operative appearance of the case; terminal ileum and sigmoid are viable. (Blue arrow indicates the terminal ileum, Yellow arrow indicates the sigmoid colon) 
component) wraps itself around the sigmoid colon (passive component).

The most common type of ISK is Type I (53.9$57.5 \%$ ), followed by Type II (18.9-20.6\%), Type III $(1.5 \%)$, and other undetermined types. The direction of torsion is clockwise in $60.9-63.2 \%$ of cases (12).

Double volvulus remains unknown when the asset liability among the different digestive segments cannot be determined. Type I and II ISK are divided into two subgroups, $\mathrm{A}$ and $\mathrm{B}$, depending on the clockwise or anticlockwise direction of the volvulus, respectively (6). Our patient had Type $1 \mathrm{~A}$ ISK.

Common symptoms and signs of ISK include abdominal pain and tenderness, asymmetrical abdominal distension, nausea and vomiting, constipation, and an empty rectum. Preoperative diagnosis is difficult (13). The accuracy of preoperative diagnosis of ISK is mostly reported to be as low as $0 \%$ to $28 \%$ due to its infrequency and atypical radiographic findings (5). Computed tomography (CT) with high spatial resolution is the gold-standard radiographic imaging modality for the evaluation of acute abdomen, as well as ISK. The safety of radiation exposure during pregnancy is a common concern; therefore, radiation exposure in pregnant women that undergo medical imaging procedures and the management of such patients are difficult issues to handle. There are radiation-related risks throughout pregnancy and these risks are related to the stage of pregnancy and the dose absorbed by the patient. To lessen these prenatal risks, the radiation dose should be minimized. The initial examination of a pregnant woman with abdominal pain should be performed with USG, which is free from radiation exposure, less expensive, and comparatively safer than CT. However, the skill level of the operator greatly influences the radiological results of the patient. Moreover, Lazarus et al. (14) found that the CT findings provided important diagnostic information in 30\% of pregnant women with abdominal pain who were determined to have normal USG findings. Efforts to avoid radiation exposure in women with acute abdomen during pregnancy may cause a diagnostic delay, especially in early pregnancy. A wrong or delayed diagnosis can pose a greater risk to the woman and foetus than any other risk associated with ionizing radiation. Prenatal doses of most properly performed procedures for the diagnosis of acute abdomen present no increased risk of prenatal death, malformation, or mental impairment (8). Therefore, in our patient, no abdominal radiography or abdominal CT were performed and the patient was primarily evaluated with USG. Moreover, the patient was operated on under emergency conditions since the vital signs observed within the last 24 hours were worsening despite a sufficient replacement therapy.

Initial management of ISK includes aggressive fluid and electrolyte resuscitation and correction of acid-base imbalance as needed. After hemodynamic stabilization, prompt surgical intervention should be performed. In addition, appropriate antibiotic therapy should be started immediately and continued for 5-7 days after the operation, which commonly includes a combination of cephalosporins, amino glycosides, and metronidazole (3).

The operative procedure to be performed in the management of ISK is dictated by the anatomical and pathological changes. Literature shows that gangrenous bowel has been encountered in 73.5$79.4 \%$ of the cases, whereas both small and large bowels have been evaluated to be viable for surgery in 20.6-26.5\% of the cases. In addition, both the small intestine and sigmoid colon have been found to be gangrenous in 52.9-60.3\% of the cases. Contradictorily, bowel gangrene has been found in $90.9 \%$ of the patients who presented within the first $24 \mathrm{~h}$ after the onset of the symptoms, as opposed to $57 \%$ of the patients who presented after the first $24 \mathrm{~h}$ (15).

Resection of the sigmoid colon is frequently advised in all cases of ISK even when it is not viable. Recurrent volvulus or repeat knotting caused by redundancy of the loop can cause gangrene following surgery. In the past, a Hartmann operation or a covering colostomy was advocated to avoid the risk official leak from colonic anastomosis. However, recent data suggest that primary colonic anastomosis could be performed safely when the history is short and the remaining bowel is clean, well vascularised, and unintended. Intraoperative colonic irrigation followed by resection and primary anastomosis is another alternative. Avoiding a colostomy is always welcome, since it reduces the morbidity and cost of health care. In patients without sigmoid gangrene, mesosigmoidostomy is administered by some surgeons to prevent recurrent ISK (9). In our patient, the ileum was twisted with a $360^{\circ}$ anticlockwise direction around the sigmoid colon. The mesentery of the ileum was also twisted and there was necrosis in the 10 $\mathrm{cm}$ ileum segment up to the ileocaecal junction. Sigmoid colon was viable, but it was longer than a normal meson. Therefore, in the first surgery, 
following a $150-\mathrm{cm}$ resection of the gangrenous ileum and sigmoid colon, end-to-end ileo-ileal and colo-colic anastomoses were performed. No ostomy was performed due to pregnancy and the bowel end was nonoedematous. In the second surgery, intraoperative cleaning of the abdomen was performed and the end ileostomy was opened due to a leak in the ileal anastomosis.

The reported mortality caused by ISK varies from $0 \%$ to $48 \%$ (mean, $35.5 \%$ ). The mortality figures are generally associated with the duration of symptoms, presence or absence of gangrene and the general status of the patient, involving the presence of septicemic shock. However, some of the reports suggest that the patients undergoing surgery within the first $24 \mathrm{~h}$ after the onset of the symptoms have a significantly higher incidence of mortality than those whose symptoms exceed $24 \mathrm{~h}$ (15).

In the diseases leading to bowel necrosis, such as ileosigmoid knot, prompt management of the patient is of vital importance. However, avoidance of CT radiography in pregnant patients is an obstacle to establish an early diagnosis of the patients, which caused the loss of the foetus in our patient. In the present report, we aimed to present a rare case of ISK in a 41-year-old, 28week pregnant woman under the light of relevant literature, which reminds us the maxim "Acute abdomen is full of surprise" and which gave us the opportunity to confirm that early treatment saves life.

\section{References}

1. Hirano $Y$, Hara $T$, Horichi $Y$, et al. Ileosigmoid knot: case report and CT findings. Abdom Imaging 2005; 30: 674-676.
2. Atamanalp SS. Ileosigmoid knotting: clinical appearance of 73 cases over 45.5 years. ANZ J Surg 2013; 83: 70-73.

3. Raveenthiran V. The ileosigmoid knot: new observations and changing trends. Dis Colon Rectum 2001; 44: 1196-1200.

4. Burrah R, Menon A, Pathan H, Ravikanth R, Kilpadi A. The ileosigmoid knot. Indian J Surg 2010; 72: 140-142.

5. Mandal A, Chandel V, Baig S. Ileosigmoid knot. Indian J Surg 2012; 74: 136-142.

6. Yazough I, Benhammane H, Morad O, et al. A rare cause of intestinal obstruction: ileosigmoid knot. Pan Afr Med J 2014; 19: 21.

7. Aslam T, Baloch MN, Maher M. Ileosigmoid knotting. J Coll Physicians Surg Pak 2007; 17: 166167.

8. Shimizu R, Hoshino Y, Irie H, et al. Ileosigmoid knot at week 13 of pregnancy:report of a case. Int Surg 2014; 99: 230-234.

9. Atamanalp SS, Oren D, Yildirgan MI, et al. Ileosigmoidal knotting in children: a review of nine cases. World J Surg 2007; 31: 31-35.

10. Machado NO. Ileosigmoid knot: a case report and literaturereview of 280 cases. Ann Saudi Med 2009; 29: 402-496.

11. Miller BJ, Borrowdale RC. Ileosigmoid knotting: a casereport and review. Aust NZJ Surg 1992; 62: 402404.

12. Atamanalp SS, Oren D, Basoglu M, et al. Ileosigmoid knotting: outcome in 63 patients. Dis Colon Rectum 2004; 47: 906-910.

13. Banerjee C, Mukhopadhyay M, Roy A, et al. The unusual volvulus: a five year retrospective analysis of nine cases. Indian J Surg 2014; 76: 100-103.

14. Lazarus E, Mayo-Smith WW, Mainiero MB, Spencer PK. CT in the evaluation of nontraumatic abdominal pain in pregnant women. Radiology 2007; 244: 784790.

15. Alver O, Oren D, Tireli M, Kayabasi B, Akdemis D. Ileosigmoid knotting in Turkey: review of 68 cases. Dis Colon Rectum 1993; 36: 1139-1147. 